\title{
A Taxonomy of Logic Attack Vulnerabilities in Component-based e-Commerce System
}

\author{
Faisal Nabi, Jianming Yong, Xiaohui Tao \\ School of Management and Enterprise, Information Systems Research Group \\ University of Southern Queensland, Australia
}

\begin{abstract}
This paper addresses the problem associated with a lack of clear web application related vulnerabilities taxonomies identifying and classifying two different types of vulnerability classes in the ecommerce CSB based web applications. The paper has done this by organizing the significant classification, which proposes the Classification of logical Vulnerabilities based on Design Flaws vs. Technical Vulnerabilities based on Implementation level Faults and Bugs in the e-commerce web application (Application component based software that resides in the mid-tier of the system) from the security assessment perspective for CBS based eCommerce application. The most important point is to integrate the knowledge contained in the attack patterns with boundary knowledge related to vulnerability of the target CBS based web Application e-commerce system and the potential threats. Taxonomies (Technical Vulnerability based) and the identified logical vulnerabilities attack Taxonomy).
\end{abstract}

\section{Introduction}

The Web applications are growing in popularity. The introduction of sophisticated mechanisms in the handling of asynchronous events in web browsers and the avail-ability of a number of frameworks for the rapid proto-typing of server-side components have fostered the development of new applications and the transition of "traditional" applications (e.g., mail readers) to web-based platforms. While new technologies have brought in significant advantages in terms of support to the development process, improved performance, and in-creased interoperability, little has been done to tackle security issues. Therefore, as the complexity of web ap-plications increase, the possibility for abuse increases as well. For example, a simple analysis of the CVE vulnerability database shows that the percentage of web-based attacks rose from $25 \%$ of the total number of entries in 2014 to $61 \%$ in 2018.This situation is made worse by the fact that web applications are usually reachable through firewalls by design, and, in addition, the server-side logic is often developed under time-to-market pressure by developers with insufficient secure software skills. As a result, vulnerable web applications are deployed and made available to the whole Internet, creating easily exploitable.

Security engineering has historically emphasized the use of industry best practices (e.g., Firewalls, Encryption (SSL/TSL), Intrusion Detection Systems, etc) as well as performing vulnerability analysis and security (e.g., penetration, white Box and Black Box) testing of existing systems to ensure adequate security. Most books and articles on security do not provide much content regarding e-commerce system security requirements, and what little that is published tends to emphasize the specification of ambiguous security goals or else focuses on architectural constraints. Rarely is either the required amount of a specific type of security specified or the security ramifications of non-security requirements addressed in security processes [1]. Cyber-attacks make up the core of any security assessment of Web based e-Commerce systems. One of the more promising research fields in this context is related to the representation of the Vulnerabilities, Attack and its patterns Classification. Several models proposed to represent them; these models usually provide a generic representation of attacks [2]. Conversely, the experience shows that attack profiles are strongly dependent upon several boundary conditions. This paper addresses the problem associated with a lack of clear web application related vulnerabilities and taxonomies identifying and classifying two different types of vulnerability classes in the e-commerce CSB based web applications. This is done by organizing the significant classification, which proposes the Classification of logical Vulnerabilities based on Design Flaws vs. Technical Vulnerabilities based on Implementation level Faults and Bugs in the ecommerce web application (Application component based software that resides within the middle-tier of the system) from the security assessment perspective of the CBS based e-Commerce application, it is necessary to integrate the knowledge contained in the attack patterns with boundary knowledge related to vulnerability of the target CBS based web Application e-commerce systems and any potential threats. Our re-search methodology focuses on a classification that separates or orders of main objects (or specimens) into classes. Classifications can be generated by a priori (i.e. non-empirically from an 
abstract model) or a Posteriori Empirically by looking at the CVE vulnerability database for security breach cases.

In this section we give some preliminary definitions related to security concepts and an overview of the risk assessment methodology we adopted as reference.

\section{Security Definitions}

We believe that in light of our research results a risk assessment in e-commerce CBS based web applications and systems are strongly connected with some concepts traditionally derived from the field of computer security, the "Five Pillars". These attributes /elements need to be defined as: the concepts of Threat, Vulnerability, Attack, Risk and Asset [3].

Threat is an actor or an Agent that is the source of danger to the system under consideration or the assets to which it has access. The threat can be a person that abuses the software, a programme running on a compromised system, or even a nonsentient event such as a hardware failure. A threat exploits vulnerability in software to attack it [5].

Vulnerability is "a flaw or weakness in the architectural design / implementation, operation, or management of an application or a service that could be exploited," according to the Internet Engineering Task Force's (IETF) Internet Security Glossary. Resulting in a loss of confidentiality, integrity, or availability of an ap-plication or resource, which leads to an undesirable consequence for system owners [4].

Attack can be identified as the entire process allowing a Threat Agent to exploit a system using one or more Vulnerabilities.

Risk may be defined as the probability that a damaging incident is happening (when a threat occurs because of a vulnerability), times the potential damage.

Asset defined as something that has value to the relevant stakeholders.

In summary - An asset is " 1 " somewhat having a relevance for an organization that " 2 "is the target of a threat age nt which, " 3 " through the use of vulnerabilities, put in act " 4 " an attack in order to "5" damage the asset and indirectly the organization.

\section{Research Methodology}

As stated in the introduction, our main objective is to develop the taxonomy of Logical Vulnerabilities in the application layer of e-commerce distributed systems which are based on multi-tiers. In various papers and texts several security assessment methodologies conceived for the security analysis of information communication technology infrastructure providing a launch pad into to an ecommerce system exist. We have selected as a reference, the work of Masera and Nai Methodology [6] in order to support our methodology. In the methodology proposed by Masera and Nai, the authors present a risk assessment methodology tailored to the analysis of the information communication technology infrastructure of complex industrial systems. This methodology focuses on the fact that in order to assess the security of a system, it is necessary to first provide a description of the system itself, its components, assets, the interaction and relationships among the components as well as, the assets and external world. Such a description could be used to identify in a systematic way the vulnerabilities affecting the whole system.

Our research methodology is also based on a Posteriori Empirically by looking at the data of CVE vulnerability databases from different levels of ecommerce type of web-based applications and systems (B2B) and (B2c) from 2002 to 2017. With a set of taxonomic characters that satisfy the criteria for the classification arbitrary selections are being used groupings of individuals on a single characteristic. These are the simplest classification schemes and require that individuals be grouped according to a simple selection criterion. For example, grouping programs by their programming language, or their use or non-use of cryptography, etc. From the analysis of this information, one can derive the evaluation of the possible damages to the components, their propagation to the system and the consequent attack patterns.

As we have stated above in the beginning about the e-commerce CBS based web applications and systems are strongly connected with some concepts traditionally de-rived from the field of computer security, in particular the "Five Pillars" are the elements, which provide strong ground to our methodology approach. On the base, we have designed Security Vulnerability Assessment Model for e-commerce CBS based web Software applications and systems that are based on Computer Security main attributes "Five Pillars" also called Computer Security elements. This will be helpful to find vulnerability, attack patterns that refers to our main objective to classify and characterizing the Logical Vulnerabilities in the application layer which is because of Design and Architectural level Flaw.

On the other hand, technical vulnerabilities as a cause of Error, Faults and Bugs at the stage of implementation level mistakes and error refer to the coding of a programme for software development. While this can be patched. Design Flaws, however, are hard to be fixed or detect by any vulnerability tools or web application code scanning tools. Therefore, no Taxonomy ever proposes any detail about the logical vulnerabilities in the application layer, which targets attack and their patterns related to the birth of business application logic vulnerabilities and attacks in the Mid-Tier (n-tier ecommerce system). 
Therefore, we propose the (SVAM) " Security Vulnerability Assessment Model in e-commerce CBS based web Software Applications and systems" based on Computer Security main attributes "Five Pillars" as stated above, which demonstrate the vulnerability life cycle and classify the main point where these vulnerabilities spread into different classes of vulnerabilities that make more subsets of vulnerabilities like Technical and Logical, both have their own weakest links that refer to attacks which both falls into different classes of vulnerabilities.

\subsection{Design Method of (SVAM)}

In order to design the above mentioned (SVAM) Model, we used the two Methodologies techniques combination. First, system security engineering methodology Developed by the US National Security Agency The Mission-Oriented Risk and De-sign Analysis called Morda [6]. This methodology provides a framework for analysing complex IS risk postures. Morda combines threat, at-tack, and mission impact concepts to derive an un-biased risk metric. The methodology helps systems and security engineers discover system deficiencies and develop reasonable design strategies to build stronger systems.

Morda uses an attack-tree analysis analogous to fault-tree analysis. Moore [7] describing and illustrating an approach for documenting attacks on software systems using attack tree information in a structured and reusable form. Analysts can then use the approach to document and identify commonly occur-ring Attack Patterns and then modify attack trees to enhance security development. Attack trees have existed in various forms, and under various names, for many years, but have been most recently described as a systematic method to characterize system security based on varying attack cycle.

The technique in our (SVAM) model which also in cooperates with the concept Attack trees has found a way to practice because they have proved to be an intuitive aid in above mentioned two different vulnerabilities life cycle threat modelled to analysis security weakest component, their apparent simplicity.

\section{Taxonomy and Classification}

Taxonomy is the theoretical study of classification, including its bases, principles, procedures and rules [11][10][8][9]. A classification is the separation or ordering of objects (or specimens) into classes. Classifications that are created non-empirically are called priori classifications. Classifications that are created empirically by looking at the data are called Posteriori Classifications.

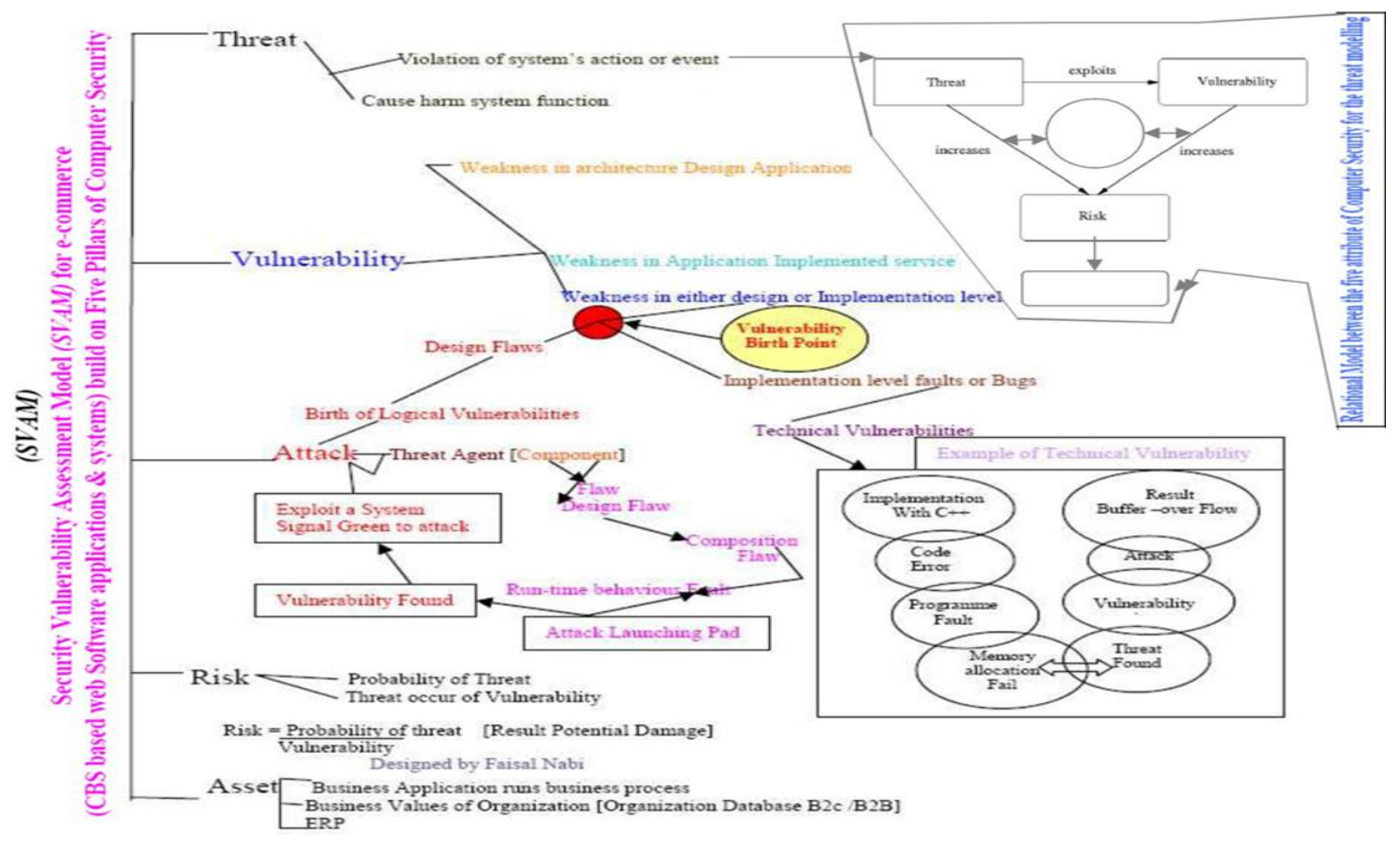

Figure 1. SVAM Model 


\subsection{Objects, Attributes and Constraints of a System}

Object: An object is an "entity" that contains or receives information, that has a unique name, and that has a set of operations that can be carried out on it [18].

- Attribute of Object of an object is a data component of an object. A derived attribute of another attribute is a data component of the later attribute.

- Property of Attribute is a characteristic of the attribute that can be derived from the attribute through the application of a function to the attribute.

- Attribute refinement is a finite refinement of attributes within larger attributes, and results in the identification of the attributes about which assumptions are made. The at-tribute refinement cannot contain a property of an attribute. Attribute refinement cannot contain a property of an attribute.

- Attribute Constraint identifies the property or set of properties that are being assumed about that attribute.

\subsection{Taxonomic Characters, Object Attributes or Features}

The bases of the development of successful classifications are taxonomic characters [12][16]. These are the properties or characteristics of the objects that will be classified. Taxonomic characters are also commonly called features, at-tributes or characteristics. [12] argues that such properties should be readily and objectively observable from the objects in question.

\section{Concept of Attack Pattern}

An attack pattern is an abstraction mechanism for de-scribing how a type of observed attack is executed. Following the pattern paradigm, it also provides a description of the context where it is applicable and then, unlike typical patterns, it gives recommended methods of mitigating the attack. In short, an attack pattern is a blueprint for an exploit. An attack pattern should typically include the following information.

In the light of the above theory and concepts, discussion and references regarding taxonomic classification of system object, attributes, properties, features and constraints based on principles, procedures and rules. We would like to first define clear definition of web software application vulnerability before moving towards the contribution of taxonomy based on classification and characterization of two different categories of vulnerabilities (Technical vs Logical).

\subsection{Web Software Application Vulnerability}

We define web software application vulnerability as "Web software application vulnerability includes mis-matches between application software Architectural / De-sign logic and the assumptions about the environment made during the development/Implementation (code writing) and operation of the programme and the environment in which the programme executes"[25].

\subsection{Taxonomy of Computer Program Security Flaws}

A Flaw can be categorized as malicious or nonmalicious.

- Malicious Flaws: are intentionally introduced in the system to cause a security violation. These take the form of viruses, worms, Trojan horses, time bombs, and trap doors in the code [17].

- Non-malicious Flaws are introduced because of missing requirements or misunderstanding of Design Logic.

Flaws are software problems that exist in the software's design. A flaw may or may not represent vulnerability in the underlying software. Mitigating a flaw typically involves significantly more effort than simply modifying a few lines of code. The problem does not lie solely in the implementation; the underlying design is flawed, there-fore, any implementation that follows the design would contain the Flaw. For example, performing sensitive business logic in an untrusted client application is a de-sign flaw that cannot mitigated by a simple measure such as modifying array bounds [19][23]. The Flaws are categorized according to the time in the soft-ware life cycle that they were introduced into the system. Time of introduction includes Flaws that were introduced during development, maintenance, or operation.

\subsection{A Taxonomy of Security Faults:}

Several security fault classification schemes have been proposed that categorized faults according to different criteria [24][26].

- Coding faults are comprised of faults that were introduced during software development. These faults could have been introduced because of errors in programming logic, as well as missing or incorrect requirements. 
- Operational faults result from improper installation of software. Most policy errors can be classified as operational faults [26].

- Environment faults result when a programmer fails to completely understand the limitations of the run-time environment or interactions between functionally correct modules [24].

\subsection{A Taxonomy of Security Error, Faults, and Failures [15]}

- An error is a mistake made by a developer. It might be a typographical error, a misreading of a specifications, a misunderstanding of what a subroutine does, etc.

- Faults are in the text of the program. "An error might lead to one or more faults" a fault is the difference between the incorrect program and the correct version.

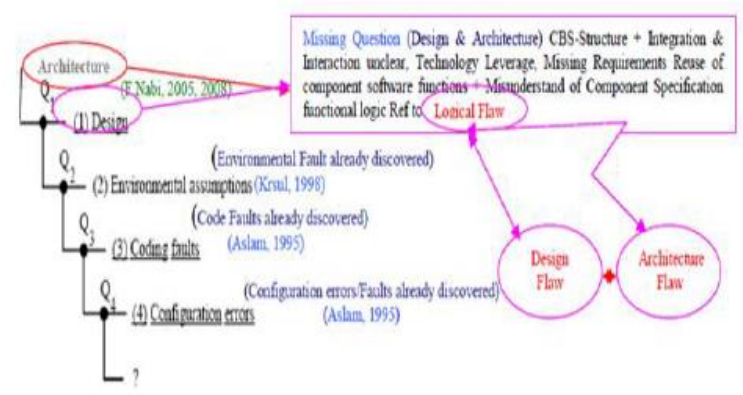

Figure 2. Taxonomy of Software Vulnerability Causes

- The execution of faulty code may lead to zero or more failures, where a failure is the [non-empty] difference between the results of the incorrect and correct pro-gram.

\section{Prior Vulnerability Taxonomies Classifications}

A taxonomy of recurring vulnerabilities may be helpful in organizing the information needed to increase security awareness. An advanced knowledge of vulnerabilities may be helpful in identifying potential attacks on a web application software before it is released to customers.

We examined 25 taxonomies from 1974 to 2017 and evaluated different level of vulnerabilities, ecommerce threat classifications property taxonomies, web application vulnerabilities, network vulnerability taxonomy and software vulnerability taxonomy before limiting the main scope of this study as focusing on logic attack problems .This is because of mismatch between design / Architecture design flaw, while developing web software application. Our attack patterns are more specific to what method can pinpoint vulnerability in a system design.

\subsection{Classification of Security Threats in e- Commerce}

In general, categorizing a phenomenon makes systematic studies possible. In particular, an organized classification of threats to e-commerce can help managers build systems that are less vulnerable [14]. An established classification would also be useful when reporting incidents to incident response teams.it is recommended the following properties for the classification for information security:

- The categories should be mutually exclusive (every specimen should fit in at most one category) and collectively exhaustive (every specimen should fit in at least one category).

- Every category should be accompanied by clear and unambiguous criteria defining what specimens are to be placed in that category.

- The taxonomy should be comprehensible and useful not only to experts in security but also to users and administrators with less knowledge and experience.

- The terminology of the taxonomy should comply with established security terminology (something that is not always easy to define).

\subsection{Classification of Web Taxonomy}

Vanden Berghe and Riordan [13] discussed a methodology for taxonomizing vulnerability and provide an example of web services, WS architectural four components model and their connections. It discusses two subclasses of "In-put Format and Input" Origin then conclude with attack flows based on boundary condition error category as example error caused by a buffer reserved memory being exceeded by unexpectedly long "Input" which execute arbitrary code by an attacker (Programme written in $\mathrm{C}$ or $\mathrm{C}++$ ). The author's Result Matrix which was proposed, gives the same as and almost a copy of Aslam,1995 and Krsul,1998's classifications [13].

Alvares and Slobodan introduced the taxonomy for web attacks. Entry point, target, HTTP Verbs, and HTTP Headers are web-specific categories that they consider important for an accurate classification of web attacks, these are not covered by general taxonomies.

In addition, some categories that can also be met in general taxonomies, such as vulnerability, and web-specific values (e.g. Code injection, HTML manipulation, canonicalization, overflows and misconfiguration). Alvares separated and ordered the taxonomy with the attacker's point of view. An attacker can get access as a result of two vulnerability errors point that may be web server or web application entry points searching for attack 
[14]. This is incomplete taxonomy and cannot be called a classification scheme,

It is more likely representing the generalize life cycle HTTP based attacking steps by a hacker that it might take. Since it fails to classify the attack patterns, vulnerabilities classification and their characterization according to their attributes, according to the classification and characterization methods referenced by Krsul [24] and Aslam [26].

\section{Taxonomy of Logic Attacks-Types and Classification}

The different types of logic Attacks occur each time since they must exploit a function or a feature that is specific to the application. The Logical Attacks focus on the exploitation of a web application's logic flow. Application logic is the expected procedural flow used in order to perform a certain action. Password recovery, account registration, auction bidding, and e-Commerce purchases are all examples of application logic. A web site may require a user to correctly perform a specific multi- step process to complete a particular action. An attacker may be able to circum-vent or misuse these features to harm a web site or its users [25]. As mentioned above the main scope of this study is to focus on "application logic-based vulnerabilities" problem that is because of a mismatch between design and Architecture while developing web software application. We have identified seven vulnerabilities in the application logic and then Taxonomy is supported by a Case as reference cause of classification design flaw.

The proposed contribution of the taxonomy is characterized by attack pattern and target agent in each kind of attack as mentioned above application logic graph-based attack pattern method, vulnerability class is logical. This is then further put into attack pattern technique to classify each vulnerability in the light of attack method, such classifications are characterized in groups of attacking parameters which defines nature of vulnerability.

\subsection{Case as a Reference: Mars Polar Landing Mission}

The case as a reference is given to study here for component-based systems and its applications. The case dis-cusses the one of the mentioned classifications of fault or defect in system composition while component-based approach is being adopted for mission critical system development by NASA [25].

Reason of Project Failure: Touchdown Monitor (TDM) (Touchdown Monitor) component failed to meet the requirement Specification as compare to its functional specification based on design by contract interface driven specification integration, which gave birth to design flaw in the MPL system and mission Failed.

Requirement Modeled of TDM: TDM is a software component of MPL system that monitor the state of three landing Legs during Two stages of descent.

Logical Component Information Processing: Multi-Tasking executive Calls TDM module at a rate of 100 times per second, receives information on the leg sensors from a second module. These two modules establish interface to TDM. During First stage, starting $5 \mathrm{KM}$ above Mars Surface, TDM software Monitors the three touchdown Legs.

Application Logic of Component: At First Stage start reading at approximately $5 \mathrm{Km}$ above Mars surface, TDM monitors touchdown legs, one sensor each leg to determine touchdown.

Processing Logic design: Developer assumed when Legs lock into deployed position, it was a known possibility sensor might indicate an erroneous touchdown signal. TDM software was to handle this potential event by Marking Leg that generates a spurious signal on two consecutive sensor-reads as having a bad sensor.

Second Stage: Starts about 40 meters above surface, TDM was to monitor the remaining Good sensor. When a sensor had 2 consecutive reads indicating Touchdown, TDM software was to command the descent Engine to Shut down.

What happened? One or more sensor did have 2 Consecutive reads be-fore40 meter Point, Leg-sensor information was stored in TDM Component Memory. When MPL crossed 40-meter point, TDM changed states and read the memory associate with the leg-sensor during first stage of descent. Result shutdown Engine.

Scientific Justification: Developer could have designed and implemented the requirement in many ways, but the Essence of design flaw, is components predicates (pre-condition, Post-condition and Invariants) violated an execution of state of bad sensor information retained by the programme variables.

It has been proved that problem was not in the implementation logic but rather design by contract technique of application logic related to logical component, and its Requirement Specification as compare to Functional, Specification based interface driven integration, which gave birth to design flaw in the MPL system and mission Failed. Therefore, classification of this defect is characterized design flaw, which is a logical defect, as identified by our classification of vulnerability through SVAM (Figure $1)$. 


\subsection{Taxonomy of Logical Vulnerabilities vs Technical Vulnerability}

In the light of our research, we would like to propose a classification and characterization of above defined two categories of vulnerability issues /problems (Technical vs Logical Vulnerabilities). These are classified on the base of their attack method as mentioned above in the classification of each vulnerability (attack pattern technique). Therefore, keeping view classification of two different categories of vulnerabilities, we have drawn a classification tree all sub-class attack under each class of vulnerability. A new Taxonomy in the application layer of e-commerce systems is depicted here with detailed classification and having characterized by their unique signature of identity.

\subsection{Vulnerability Life Cycle in Context Software Process Model}

Attack patterns are descriptions of common methods for exploiting software. They are derived from the concept of design patterns [46] the proposed model clearly depicts the stages of two different vulnerabilities life cycle as mentioned in the figure 6.One explains Design and architecture and other one shows Implement level, each phase shows two different vulnerability causes, such as design phase refers to design flaw and architectural flaw and Implementation phase shows faults, bugs and errors. The technique of identifying vulnerabilities is achieved via mismatching a sequence of components in a system de-sign that permits the sequence of events in the attack pattern to occur. If it exists, then the vulnerability may exist in the application being analysed. The proposed model also provide a detailed information of all stages of secure system development process and identifies the two different categories of vulnerabilities, both at design and implementation level. This helps to understand two different vulnerabilities lifecycle and there point of close-ness as mentioned in figure.

\section{Conclusion}

A taxonomy is a footprint for software designers to per-form secure system engineering. The approach taken in this paper focus on component-based web ecommerce applications and logical vulnerabilities to characterize and then make classification of the vulnerabilities. There-fore, by performing the proposed approach and method in the design phases increases security awareness at the be-ginning of the software process and encourages risk management to begin early so that security team be able determine how to fortify their software application logic. We have also classified the two different categories of vulnerability in component based software development model and depict the birth of attack design by cause of vulnerability into different phases of development life cycle, which is helpful for developers during the software design adoption of security by design technique.

\section{References}

[1] Firesmith D. G., (2007), A taxonomy of security related requirements, software engineering institute, Carnegie Mellon University.

[2] Schneier, B., Secrets and Lies: Digital Security in a Networked World, John Wiley and Sons, August 2000.

[3] Jones, A., Ashenden, D., (2005), Risk Management for Computer Security: Protecting Your Network and Information Assets, March, Elsevier.

[4] Bishop, M., (2004), Computer Security Art and Science, November, Addison Wesley.

[5] Masera, M., Nai Fovino, I., and Sgnaolin, R., (2005), A Framework for the Security Assessment of Remote Control Ap-plications of Critical Infrastructure. ESReDA $29^{\text {th }}$ Seminar, Ispra.

[6] US National Security Agency (2005), The MissionOriented Risk and Design Analysis called (Morda).

[7] Moore, A., Ellison, R., and Linger, R., (2001), "Attack Modelling for Information Security and Survivability," Technical Note, CMU/SEI-2001-TN-001, Software Engineering Institute, Carnegie Mellon University, March.

[8] Grolier Incorporated. (1993), Encyclopaedia Americana, Deluxe Library Edition, Grolier Inc.

[9] EBRIT, (1997), Britannica Online version 97.1.1. http://www.britannica.com (Access Date: 11 December 2018).

[10] WEBOL (1998), Merriam-Webster Online: WWWebster Dictionary. http://www.m-w.com/dictionary. htm (Access Date: 15 November 2018).

[11] Simpson, G.G., (1945), The principles of classification and a classification of mammals. Bull. Am. Mus. Nat. Hist. 85: i-xvi, 1-350.

[12] Simpson, G. G., (1961), Principles of Animal Taxonomy. Columbia University Press.

[13] Vanden Berghe, C., and Riordan, J., (2005), A taxonomy methodology applied to web services, IBM Zurich Research Laboratory.

[14] Alvares and Slobodan, (2003), A taxonomy of web attacks, ICWE, 2003 LNCS 2722, Spriger-Verlag Berlin Heidelberg.

[15] IEEE. (1990), ANSI/IEEE Standard Glossary of Software Engineering Terminology, IEEE Press.

[16] Glass, R. L., and Vessey, I., (1995), Contemporary Application-Domain Taxonomies. IEEE Software 12, 4 (July), 63,76

[17] Carl Landwher et al. (1993), A taxonomy of computer program security flaw Technical report, Naval Research Laboratory, November. 
[18] Longley, D., and Shain, M., (1990), The Data and Computer Security Dictionary of Standards, Concepts, and Terms. Macmillan Stockton Press.

[19] Faisal Nabi, (2005), "secure business application logic for e-commerce systems" Journal computers and Security.

[20] McPhee. W. S., (1974), "Operating System Integrity in OS/VS2.IBM Sys. J.”, vol. 13, no. 3, pp. 230-52.

[21] Abbott, R. P., et al, (1976), "Security Analysis and Enhancements of Computer Operating Systems," Report NBSIR 76- 1041, Institute for Computer Science and Technology, Natl. Bur. of Stnds, Apr.

[22] Bisbey, R., and Hollingsworth, D., (1978), "Protection Analysis Project Final Report", Information Sciences Institute, University of Southern California, Marina Del Rey, CA.

[23] Nabi, F., (2011) "Desgning secure framework method for e-commerce systems" Journal of Network Security.

[24] Krsul, (1998), "Software Vulnerability Analysis", $\mathrm{PhD}$, Purdue University, West Lafayette.

[25] Nabi, F., (2017), A Process of Security Assurance Properties. Unification for Application Logic, International Journal of Electronics and Information Engineering, Vol.6, No.1, pp.40-48, Mar.

[26] Aslam, T., (1995), "A taxonomy of Security Faults in the Unix Operating System," M.S. Thesis, Purdue University.

[27] Bishop, M., (1995), “A Taxonomy of UNIX System and Network Vulnerabilities," Technical Report CSE-9510, Purdue University, May.

[28] Du W., and Mathur. A. P., (2000), "Testing for software vulnerability using environment perturbation", Proceeding the International Conference on Dependable Systems and Networks (DSN 2000), Workshop on Depend-ability versus Malicious faults, http://www.ceria s.pur-due.edu/homes/duw/research/paper/ftcs30 workshop. (Access Date: 19 Deceember 2018).

[29] Lough, D., (2001), "A Taxonomy of Computer Attacks with Applications to Wireless Networks," PhD thesis, Virginia Polytechnic Institute and State University.

[30] Piessens, F., (2002), "A taxonomy of causes of software vulnerabilities in internet software", Supplementary Proceedings of the 13th International Symposium on Software Reliability Engineering.

[31] Gray, (2003), "An Historical Perspective of Software Vulnerability Management,” Info. Sec. Tech. Rep., vol. 8, no. 4, April, pp. 34-44.

[32] Jiwnani K., and Zelkowitz, M., (2004), "Susceptibility Matrix: A New Aid to Software Auditing," IEEE Sec. and Privacy, vol. 2, no. 2, Mar-Apr, pp.16-21.

[33] Pothamsetty, V., Akyol, B., (2014), “A Vulnerability Taxonomy for Network Protocols: Corresponding Engineering Best Practice Countermeasures," in IASTED Int. Conf. on Commun., Internet, and Inform. Tech. (CIIT), ACTA Press, US Virgin Islands.
[34] Tsipenyuk, K., Chess, B., and McGraw, G., (2005), "Seven Pernicious Kingdoms: A Taxonomy of Software Security Errors," IEEE Sec. and Privacy, vol. 3, no. 6, Nov.- Dec. pp. 81-84.

[35] Weber, S., Karger, P. A., and Paradkar. A., (2005), “A Soft-ware Flaw Taxonomy. Aiming Tools", At Security Software Engineering for Secure Systems-Building Trustworthy Applications (SESS'05).

[36] Seacord, R. C., "Secure Coding in C and C++". USA: Addison-Wesley Professional, 2005.

[37] Hansman, S., Hunt R., (2005), "A taxonomy of network and computer attacks". Computer and Security, vol. 24, Issue 1, February, pp. 31-43.

[38] Kjaerland, M., (2006), "A taxonomy and comparison of computer security incidents from the commercial and government sectors". Computers and Security, Volume 25, Issue 7, October, pp 522-538.

[39] FORTIFY, http://www.fortifysoftware.com/ (Access Date: 10 November 2018).

[40] Bazaz, A., Arthur, J. D., (2007), "Towards a Taxonomy of Vulnerabilities", HICSS, 2007, Proceedings of the 40th Annual Hawaii International Conference on System Sciences, pp. 163a, doi:10.1109/HICSS.2007.566.

[41] Igure V. M. and R. D. Williams, (2008), "Taxonomies of Attacks and vulnerabilities in Computer Systems", IEEE Communications Surveys and Tutorials 1st Quarter.

[42] Simmons, C., Ellis, C., Shiva, S., Dasgupta, D., and Wu, Q. (2009), “AVOIDIT: A Cyber Attack Taxonomy", University of Memphis, Technical Report CS-09-003.

[43] Cebula, J. J., and Lisa, R. Y. (2010), “A Taxonomy of Operational Cyber Security Risks", (Carnegie Mellon University / Software Engineering Institute No. CMU/SEI2010- TN-028). http://www.sei.cmu.edu/library/abstracts /report s/10tn028.cfm (Access Date: 10 November 2018).

[44] Scott, D., and Angelos, S., (2013), "Towards a Cyber Conflict Taxonomy", 5th International Conference on Cyber Conflict K. Podins, J. Stinissen, M. Maybaum (Eds.).

[45] Joshi C., and Singh. U. K., (2014), "ADMIT- A FiveDimensional Approach towards Standardization of Network and Computer Attack Taxonomies". International Journal of Computer Applications 100(5):30-36, August.

[46] Johnson, R., Gamma, E., Vlissides, J., Helm, R., (1995), De-sign Pattern: Reusable Object-Oriented Software, pp 65-75, Addition Wesley. 